centralis medialis, Nucl. ventralis medialis, Nucl. ventralis posteromedialis (VPM), and other paramedian nuclei in the thalamus.

When EEG exhibited arousal pattern, the response in CM-Pf complex could not be identified by the single stimulus, but it was obtained by double stimuli (1.0 msec in duration, in an interval of $2-3 \mathrm{msec}$ ) delivered to the vagus nerve. On this occasion, high frequency stimulation $(10 \mathrm{cps}$.) of the vagus nerve caused spindle bursts in $\mathrm{CM}$ only during tetanic stimulation.

Under light Nembutal anesthesia, on the other hand, single and double stimuli equally produced evoked responses to vagal stimulation, although the amplitude was exhibited in larger magnitude by double stimuli than by single.

After decortication, potentials evoked in CM-Pf complex by stimulation of the vagus became much pronounced, though double stimuli produced more intensive responses also on this occasion.

Phase reversal of the evoked potential was observed in CM as well as VPM. The sciatic and vagal responses reversed their phases on the different level in VPM, but on the same level in CM-Pf complex.

Alternative occlusive interaction between potentials evoked by the stimulation of the vagal and sciatic nerves was observed in CM, but not in VPM. In CM, the potential to the sciatic stimulation exhibited dominant occlusive effect over the potential to the vagal stimulation.

These results seem to suggest that the cerebral cortex might exert tonic interference upon afferent vagal impulses which ascend to $\mathrm{CM}$, and this corticofugal inhibitory effect might be released by light Nembutal anesthesia.

\title{
131. Pain and C-Fiber (1)
}

\author{
K. Tsunekawa, A. MachizuKa and K. Kumada
}

2nd Surgical Division, Kyoto University Medical School

1. i) The saphenous nerve was severed in the dog and reunited by tubulation with an arterial tube.

Conduction velocity and spike duration was measured in vivo on both the operated and unoperated sides in ten animals 50 to 100 days after operation.

ii) The earlier, the more the affinity between the regenerated and the C-fiber was shown.

2. i) An observation was made on the sensory impulses in the cat's visceral

$$
-173-
$$


nerve of intestine by intraarterial injection of Acetylcholine to the mesenteric artery.

ii) Such stimuli was usually painful and found to excite the numerous C-fibers and the bulk of discharge was found in the ascending phase of the inner pressure curve of intestine as also in the abdominal muscles.

iii) There was no distinguishing feature of the intestinal E.M.G. in spite of usual pain response.

iv) In the E.M.G. of both the intestine and abdominal muscles, a few discharge was fonnd at the descending phase of the inner pressure curve.

v) Anoxia of intestinal tissue at the maximal contraction and electromyographical feature was not found to attribute to excitation of $\mathrm{C}$-fiber by painful stimuli with intra-arterial injection of acetylcholine.

\title{
132. On the Mechanism of Lateral Nuclei of the Thalamus.- especially on the Interrelationship with the Cerebellar Dentate Nucleus and Cerebral Motor Cortex
}

\author{
M. Yoshida, I. Hirota and M. Uno
}

Dept. of Psychiatry, Tokyo University

\section{Physioanatomical Study of the Forel-H Field}

\author{
Jiro MuKawa \\ Department of Neurosurgery, Osaka University Medical School
}

An experimental study was attempted on 42 cats to make an analysis of the effect of subcortical and brain stem lesion on the Metrazol-induced corticogenic epileptic convulsion based on EEG-discharge and EMG-convulsion as indicators. Anatomical study was also made to follow the degeneration of fibers by means of Marchi technique in cat after making a lesion in the Forel-H field.

1. A definite threshold increment of eliciting the seizure was fonnd in the case of bilateral lesioning of the Forel-H field. In contrast, no change in the threshold was found in case of bilateral lesioning of the other parts 